\title{
Effect of the Lead Dimensionality Over Transport Properties in Quantum Dots
}

\author{
L. Craco ${ }^{1}$ and G. Cuniberti ${ }^{2}$ \\ ${ }^{1}$ Instituto de Física "Gleb Wataghin", Unicamp, 13.081-970 Campinas, SP, Brazil \\ ${ }^{2}$ Max-Planck-Institut für Physik komplexer Systeme, Nöthnitzer Str. 38, D-01187 Dresden, Germany
}

Received on 23 April, 2001

\begin{abstract}
We theoretically investigate the effect of the lead dimensionality on the non-equilibrium electron transport through quantum dots. More precisely, we study nonlinear transport in a quantum dot coupled to leads of diverse dimensionality. We show that the presence of the latter strongly determines the resulting transport properties. Differently from higher dimensional leads (wide and smooth band limit), van Hove singularities in the density of states of low-dimensional reservoirs determine sharp resonances in the differential conductance at finite applied voltages as well as in the dot spectral density. It is also shown that, due to the finiteness of the terminal bandwidth, the differential conductance change its sign at higher biases. These results clearly indicate that the environment does play an important rôle in determining transport properties in mesoscopic systems.
\end{abstract}

Quantum dots represent perhaps the ultimate limit in the design of miniaturized electronic circuits. Transport measurements offered a wide variety of information for the theoretical understanding of the quantum dot physics especially as concerning to the role of single electron charging [1], spin ground states electronic correlation [2], and Kondo effect [3]. One of the unsolved issues of the foremost nanofabrication is the interfacing between quantum dots and the external environment. The byproducts of this effort can give operational criteria for the potential applicability of these structures in large scale integration. Low dimensional systems are thought as possible tools for overcoming this difficulty. Examples come from the hybridation of biological and electronic material [4], and from novel 1D-like materials such as carbon nanotubes [5]. A theoretical comprehension of the coupling between different dimensional electronic systems in transport experiments is consequently needed.

To the purpose of understanding the interplay between small dots and low dimensional reservoirs coupled to them, we consider here a single level quantum dot (QD) connected to leads of different dimensionality. We model the experimentally plausible case in which a QD is coupled to two van Hove leads (vHL), that are non interacting 1D reservoirs, and to high dimensional reservoirs with parabolic (PL) density of states (DOS) [6]. We also consider the mixed configuration where the QD is simultaneously connected to two different terminals: vHL and PL leads.

Here we treat the simplest model that takes into ac- count the resonant tunneling through a single quantum state, more precisely, the noninteracting two-orbital Anderson impurity model. This model has the advantage of being reduced in complexity so that it preserves exactly solvable features, and consequently it allows a deep investigation of the possible physical behavior in the full parameter range. Moreover, it grasps the basic physics of more sophisticated system such as Kondo systems in the strong coupling limit [7].

The noninteracting two-orbital Anderson impurity model is described by the Hamiltonian

$$
\begin{aligned}
H & =\sum_{k, \sigma, \alpha} \varepsilon_{k}^{\alpha} c_{k \sigma \alpha}^{\dagger} c_{k \sigma \alpha}+\sum_{\sigma} \varepsilon_{0} d_{\sigma}^{\dagger} d_{\sigma} \\
& +\sum_{k, \sigma, \alpha} t_{k}^{\alpha}\left(c_{k \sigma \alpha}^{\dagger} d_{\sigma}+\text { H.c. }\right)
\end{aligned}
$$

where $\varepsilon_{k}^{\alpha}$ represents the single particle energy in the reservoir $\alpha=L, R$ with their chemical potential difference being the applied voltage, that is $\mu_{\mathrm{L}}-\mu_{\mathrm{R}}=\mathrm{eV}$. The parameters $\varepsilon_{0}$ and $t_{k}^{\alpha}$ denote the single particle energy in the single level QD and the coupling between QD and reservoir states, respectively.

To make contact with experiments we calculate the current using the Landauer [8] formula

$$
I=\frac{2 e}{\hbar} \sum_{\sigma} \int d \omega \tilde{\Gamma}(\omega)\left[f_{\mathrm{L}}(\omega)-f_{\mathrm{R}}(\omega)\right] \rho_{\sigma}(\omega),
$$

where $\tilde{\Gamma}(\omega)=\Gamma_{\mathrm{L}}(\omega) \Gamma_{\mathrm{R}}(\omega) /\left(\Gamma_{\mathrm{L}}(\omega)+\Gamma_{\mathrm{R}}(\omega)\right), \Gamma_{\alpha}(\omega)=$ $\pi \sum_{k}\left|t_{k}^{\alpha}\right|^{2} \delta\left(\omega-\varepsilon_{k \alpha}\right)$ is the coupling between the $\operatorname{dot}$ 
level and the lead $\alpha$. In Eq. (2) the transmission probability span the product of $\tilde{\Gamma}(\omega)$, the Fermi function of leads $f_{\alpha}(\omega)=1 /\left(e^{\beta\left(\omega-\mu_{\alpha}\right)}+1\right)$ and the spectral density of states (DOS) of the electron in the QD: $\rho_{\sigma}(\omega)=-\frac{1}{\pi} \operatorname{Im} G_{\sigma}(\omega)$.

The retarded one-particle Green's function for the noninteracting Anderson impurity model is given by

$$
G_{\sigma}(\omega)=\frac{1}{\omega-\varepsilon_{0}-\Delta(\omega)},
$$

where $\Delta(\omega)=\sum_{\alpha} \Delta_{\alpha}(\omega)$ is the self-energy due to the tunneling into the leads, which is given by

$$
\Delta_{\alpha}(\omega)=\sum_{k \in \alpha} \frac{\left|t_{k}^{\alpha}\right|^{2}}{\omega-\varepsilon_{k}^{\alpha}} .
$$

In what follows we will assume an $k$-independent coupling between the leads and the single level QD: $t_{k}^{\alpha} \equiv t_{\alpha}=0.25 W_{\alpha}, W_{\alpha}$ is the half with of conduction band in the reservoir $\alpha$. In doing so, the $k$-dependence in Eq. (4) is restricted to the tight-binding energies $\varepsilon_{k}^{\alpha}$ and one can naturally replace the sum over $k$ by and integral in energy in the form

$$
\Delta_{\alpha}(\omega)=t_{\alpha}^{2} \int d \epsilon \frac{\rho_{0}^{\alpha}(\epsilon)}{\omega+\mu_{\alpha}-\epsilon}
$$

where $\rho_{0}^{\alpha}(\epsilon)$ is the uncorrelated DOS for the reservoirs. In the case of vHL, we consider a $1 \mathrm{D}$ system for which $\rho_{0}^{\alpha}(\epsilon)=\frac{\theta\left(W_{\alpha}-|\epsilon|\right)}{\sqrt{W_{\alpha}^{2}-\epsilon^{2}}}[9]$. To describe a system where the effect of the van Hove singularities is absent, we consider an hypercubic lattice (PL) whose orbital is described by $\rho_{0}^{\alpha}(\epsilon)=\frac{3}{4 W_{\alpha}}\left(1-\frac{\epsilon^{2}}{W_{\alpha}^{2}}\right)$ for $|\epsilon| \leq W_{\alpha}$ and zero otherwise [10].

Let us now turn our attention to the numerical results. Without loss of generality, we will consider here the symmetric $\varepsilon_{0}=0$ (zero gate voltage) case at zero temperature. The van Hove singularities observed in the density of states (DOS) of the QD at Figs. (1a) and $(1 \mathrm{~b})$ are related with the presence of the same singularities in the leads. It is well known that the singularities in a free electron 1D system appears in the border of the band. Since in the equilibrium case $e V=0 W\left(W_{\alpha} \equiv W\right)$ the bands of the left and right leads coincide, we therefore observe only two van Hove singularities in DOS of the single level quantum dot, see Fig. (1a). Once the applied voltage is considered $e V \neq O$, as in Fig. (1b), the Fermi level of the two leads are shifted from the equilibrium value and the total DOS of the external bath $\Delta(\omega)$ for the pure 1D environment presents four van Hove singularities. Since the dot feels the presence of both reservoirs simultaneously, we consequently observe four singularities for finite applied voltage. As one can see in Figs. (1a) and (1b) the spectral DOS of the dot is symmetric around the zero bias voltage for the non-mixed scenarios (vHL and PL) in both equilibrium and non-equilibrium cases. This symmetric property breaks down in the mixed configuration for $e V \neq 0$. In this regime the central peak is shifted to positive energies and due to the bias voltage the two $\mathrm{vH}$ singularities of the $1 \mathrm{D}$ reservoir are also shifted to positive energies, as one can see in Fig. (1b).
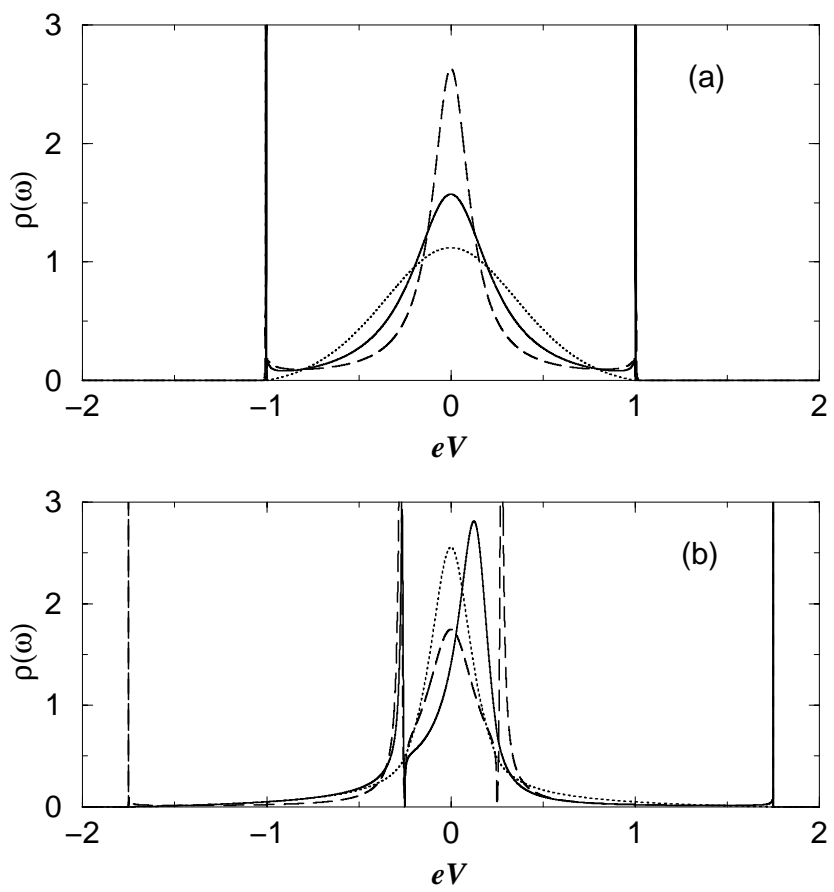

Figure 1. Spectral DOS for (a) $e V=0$ and (b) $e V=0.75$ in the three different environment configurations: parabolic leads (dotted line), 1D-leads (dashed line) and the hybrid case (solid line).

Our results for the differential conductance and the current are shown in Figs. (2a) and (2b), respectively. As expected the differential conductance is decreasing when increasing the bias voltage in the vHL, PL and mixed scenarios. Nevertheless when the bias voltage approaches the critical value $W_{\mathrm{c}} \equiv W / e$ the lead dimensionality may be revealed by nonequilibrium calculations. In fact, the presence of at least one $\mathrm{vH}$ lead induces a resonance in the differential conductance, which does not appear in the PL case, see Fig. (2a). By further increasing the bias voltage a negative differential conductance is obtained for all systems. In the case of pure vHL reservoirs, we also observe a large negative resonance at the border of the band, while for the $\mathrm{PL}$ is characterized by a suppression of $\mathrm{d} I / \mathrm{d} V$ at the same energy.

In Fig. (2b) we show our results for the current. As one can see, the current first increases up to energies near $W_{\mathrm{c}}$ and after start to decrease due to the finiteness of the lead bandwidth. The resonance observed in the differential conductance at the critical bias voltage in both vHL and mixed configurations is closely related to the sharp increasing of the current at energies just 
below $W_{\mathrm{c}}$. It is important to mention here that similar type of effect has been recently observed in currentvoltage measurements of gold point contacts coupled to (BCS) superconducting aluminum leads [11].
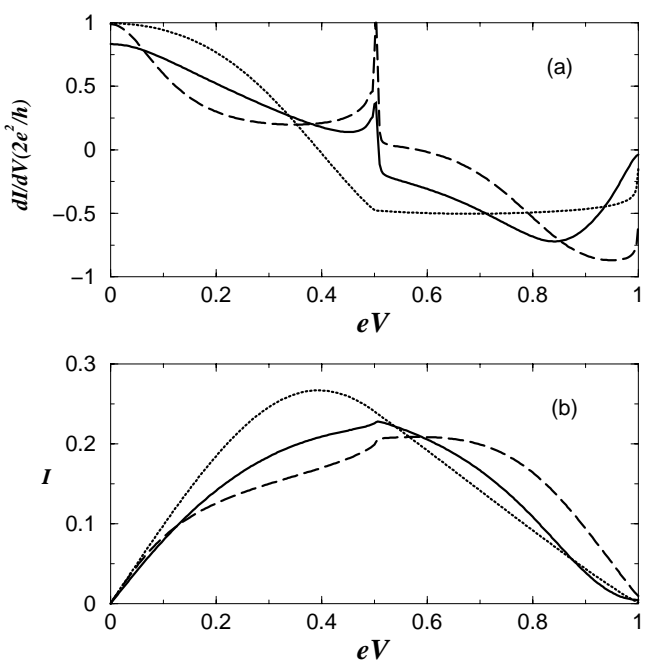

Figure 2. Differential conductance (a) and the current (b), respectively, for $e V=0$ in the three different environment configurations: parabolic leads (dotted line), 1D-leads (dashed line) and the hybrid case (solid line).

Summarizing, we have investigated the nonequilibrium electron transport through a QD connected to leads of different dimensionality. The peculiar nature of the leads is reflected in a resonant behavior of the differential conductance induced by a discontinuity in the current. Contrary to parabolic DOS leads (emerging in systems with large coordination number), the current and the differential conductance manifest abrupt changes for values of the external applied voltage which match the one-dimensional half bandwidth.

\section{Acknowledgments}

LC was also supported by the Fundação de Amparo à Pesquisa do Estado de São Paulo (FAPESP). The research of GC at MPI is sponsored by the Schloeßmann Foundation. We acknowledge the kind help of the editorial office of the Brazilian Physical Society in the edition of the Proceedings of the BWSP-10.

\section{References}

[1] Single Charge Tunneling, Vol. 294 of NATO Advanced Study Institute series B, edited by H. Grabert and M. H. Devoret (Plenum Press, New York, 1992).

[2] T. H. Oosterkamp et al., Phys. Rev. Lett. 80, 4951 (1998).

[3] D. Goldhaber-Gordon et al., Nature 391, 156 (1998); S. M. Cronenwett, T. H. Oosterkamp, and L. P. Kouwenhoven, Science 281, 540 (1998).

[4] D. Porath, A. Bezryadin, S. de Vries, and C. Dekker, Nature 403, 635 (2000); B. Crone et al., Nature 403, 521 (2000); S. S. Wong et al., Nature 394, 52 (1998).

[5] R. Saito, G. Dresselhaus, and M. S. Dresselhaus, Physical Properties of Carbon Nanotubes (World Scientific Publishing Co. Pte. Ltd., London, 1998).

[6] L. Craco and K. Kang, Phys. Rev. B 59, 12244 (1998).

[7] By using the slave boson representation with mean field approximation the solution the strongly correlated Anderson impurity model is mapped onto the noninteracting one with the hybridisation and the bound state selfconsistently renormalized, for more details see for example, G. M. Zhang et al., Phys. Rev. Lett. 86, 704 (2001).

[8] Y. Meir and N. S. Wingreen, Phys. Rev. Lett. 68, 2512 (1992).

[9] E. N. Economou, Green's Functions in Quantum Physics (Springer, Berlin, 1990).

[10] L. G. Brunet, R. M. Ribeiro Teixeira, and J. R. Iglesias, Solid State Comm. 68, 477 (1988).

[11] E. Scheer et al., Phys. Rev. Lett. 86, 284 (2001). 